\title{
A numerical model for temporal variations during explosive central vent eruptions
}

\author{
A. Folch and J. Martí \\ Institute of Earth Sciences "Jaume Almera," Consejo Superior de Investigaciones Cientificas, Barcelona, Spain \\ R. Codina and M. Vázquez \\ International Center for Numerical Methods in Engineering, Universitat Politècnica de Catalunya, Barcelona, Spain
}

\begin{abstract}
An axisymmetrical numerical model has been developed in order to find the temporal evolution of pressure, the position of the exsolution level, the velocity field, the eruption rate, and the amount of erupted material of a shallow, volatile-rich, felsic magma chamber during a Plinian central vent eruption. The overpressure necessary to trigger the eruption is assumed to result from crystallization-driven volatile oversaturation. We solve the resulting set of equations using a finite element method. The results obtained show that the pressure at the conduit entrance decreases exponentially as the eruption proceeds. This produces a shifting of the exsolution level, so that deeper parts of the chamber become progressively volatile oversaturated during the eruption. We assess the influence of chamber geometry and the physical properties of the magma on the computed parameters using several numerical examples. The results are also compared with those predicted by previous models from the literature and are found to be in good agreement with documented eruptions. The model constitutes a first attempt to numerically model the dynamics and the temporal evolution of the most relevant physical parameters during withdrawal from a closed magma chamber.
\end{abstract}

\section{Introduction}

Silicic magma is commonly stored in shallow magma chambers located a few kilometers below the surface of the Earth [Smith, 1979]. When the chamber overpressure exeeds the tensile strength of the surrounding rocks a volcanic eruption may be triggered through fractures that propagate to the surface. The increase in magma pressure necessary to trigger the eruption can be produced either by the addition of new magma into the chamber [Blake, 1981] or by the exsolution of volatiles associated with the cooling of the magma during fractional crystallization [Blake 1984; Tait et al., 1989]. We define a system as open when the eruption is due to the addition of new magma into the chamber and as closed when the triggering mechanism is crystallization-driven volatile exsolution.

A good knowledge of the formation and dynamics of magma chambers is crucial to understanding how magmas evolve and how they subsequently erupt at the Earth surface. The dynamics of magma chambers prior to and during volcanic eruptions has become a subject of increasing interest during the recent years. Several analytical [Blake, 1981; 1984; Tait et al., 1989; Bower and Woods, 1997], experimental and numerical [Spera, 1984; Spera et al., 1986; Trial et al., 1992] models have been developed to study the withdrawal of magma from a shallow magma chamber. However, the dynamics of magma chambers during volcanic eruptions are still far from well-understood.

The application of numerical simulations has become a useful tool in such studies, as they allow the variation of some of the

Copyright 1998 by the American Geophysical Union.

Paper number 98JB01671.

0148-0227/98/98JB-01671\$09.00 main physical parameters that control the dynamics of magma chambers during volcanic eruptions to be constrained. This has provided an opportunity to understand the significance of some compositional and stratigraphic variations found in pyroclastic deposits in terms of preeruptive gradients in the chamber and dynamics of the magma withdrawal process [Spera, 1984; Spera et al., 1986; Trial et al., 1992]. So far, all these numerical simulations consider the magma chamber as an open system, in which the magma withdrawal process is only driven by pressure forces associated with magma replenishment. Spera [1984] simulated a central vent eruption assuming that magma behaves as a homogeneous, Newtonian, incompressible fluid of constant viscosity. Trial et al. [1992] improved this model considering a preeruptive compositional gradient in order to account for the variations observed in erupted diposits. Overpressure produced by oversaturation of volatiles during fractional crystallization has also been proposed as a plausible mechanism of triggering explosive eruptions in felsic systems [Blake, 1984; Tait et al., 1989]. However, no attempts have been made to simulate numerically the dynamics of an erupting magma chamber in such conditions.

The aim of this paper is to characterize the evolution of the physical properties of magma inside the chamber during a Plinian eruption triggered by oversaturation of volatiles in a closed system. We develop an axisymmetrical numerical model of the interior of a chamber which is filled with a homogeneous, volatile-rich, felsic magma in which vesiculation is considered to have taken place prior to the eruption. The driving force that leads to the eruption is thus the volatile overpressure produced during magma cooling and crystallization. In order to find the pressure evolution, the evolution of the exsolution level, the velocity field in the chamber, and the mass discharge rate at the 


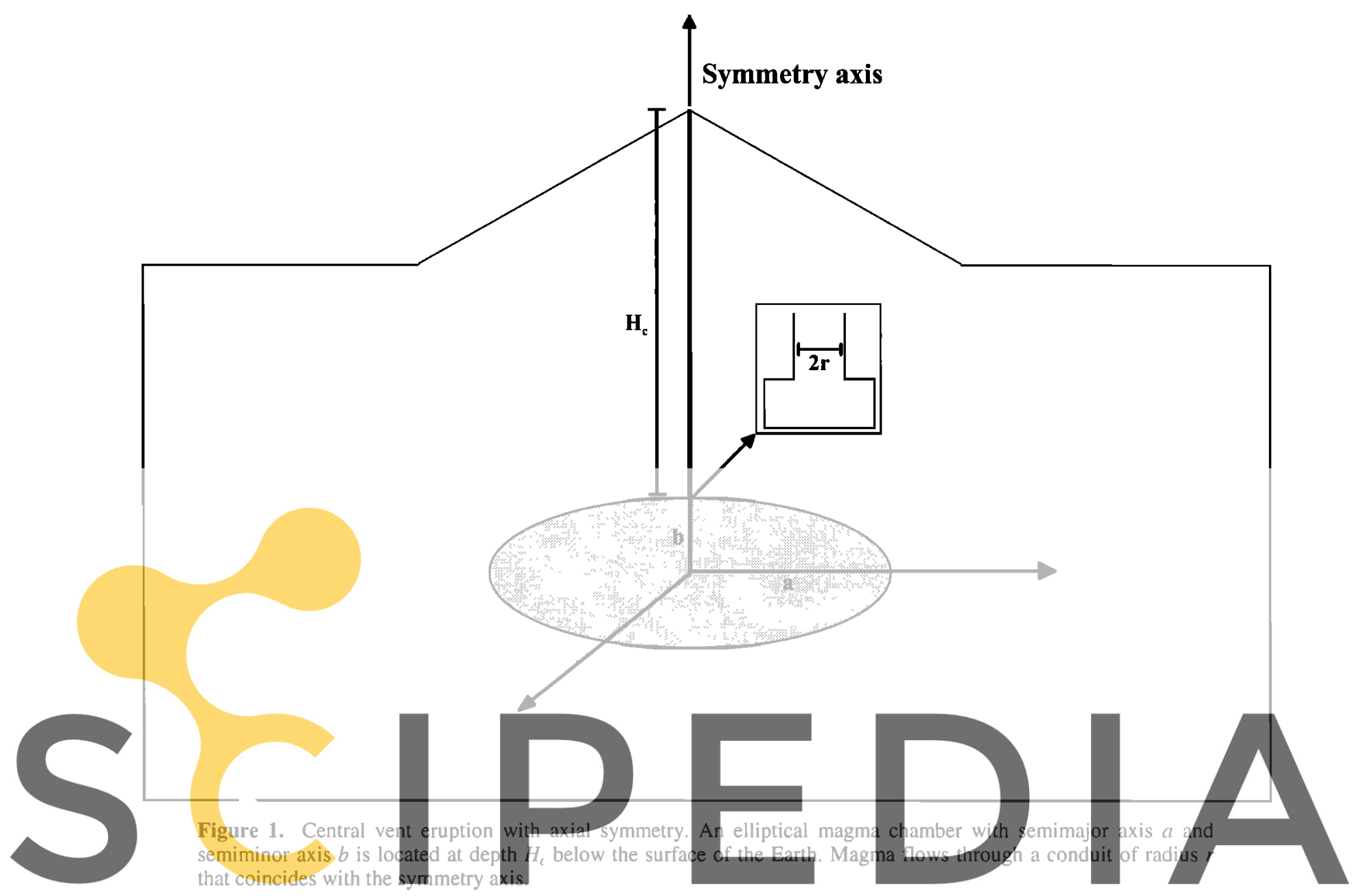

\section{Register for free at https//www.scipedia. com to} equations using a finite element method (FEM). Magma is treated as an incompressible Newtonian fluid below the exsolution level but is compressible once is vesiculated. In the domain above the exsolution level, magma is considered as a homogeneous two-phase flow, and we develope a barotropic state law, where density depends only on pressure, depending on the solubility law.

All the results obtained depend on several parameters (geometry of the chamber, chemical composition of magma, magma properties, etc.). The application of the model allows us to discuss the relative significance of each of these parameters in the eruption process. As we compute the pressure evolution inside the chamber during the withdrawal process, we can estimate the variation of the exsolution level with time. To know the temporal pressure variation at the conduit entrance it is also important to constrain models for the conduit, which generally assume either a constant pressure or a linear decrease with time. In contrast, our model indicates that pressure at the entrance of the conduit decreases exponentially with time.

\section{The Model}

\subsection{Physical Model}

We consider a central vent eruption with axial symmetry as shown in Figure 1. The domain comprises a magma chamber located at a depth $H_{\mathrm{c}}$ and a central conduit of radius $r$ along the

\section{download the version without the watermark dimensional problem in two dimensions by using cylindrical} coordinates.

Plinian eruptions involve conduit erosion during their initial stages. To avoid the coupling with this mechanical problem, we will assume that the magma chamber has rigid walls and that the conduit is not eroded by the flow. Neither the chamber nor the conduit geometries change during the eruption. We consider that the magma behaves as a Newtonian incompressible fluid below the exsolution level but is compressible above this level, where magma is assumed to be a barotropic fluid.

The problem may be approached without considering temperature because magmas have a large thermal capacity and the host rock has a low thermal conductivity so that temperature variations inside the chamber occur on timescales which are orders of magnitude greater than the duration of an eruption [Dobran, 1992]. Therefore we assume that the chamber behaves as a thermaly isolated system during the eruption. This assumption would not be so valid in modeling the conduit, where cooling of the magmatic mixture by up to $100^{\circ} \mathrm{C}$ may occur [Buresti and Casarosa, 1989].

As water is by far the most abundant gas species in felsic magmas, it is considered as the only gas phase. Water content is a parameter of the model, and its value depends upon the magma chamber depth and the lithostatic pressure. We choose a water content which ensures that the chamber is partially vesiculated prior to eruption, in order to reproduce a situation where the triggering mechanism of the eruption could be the 
volatile oversaturation. Finally, we assume a chemically homogeneous magma composition for the whole chamber. It should be said that this homogeneous composition is a major limitation of the model, because most magma chambers are zoned systems with compositional gradients [Huppert and Sparks, 1984].

\subsection{Governing Equations}

The governing equations for this problem are the NavierStokes equations, which are derived in continuum mechanics from general principles of mass, energy, and momentum conservation. The energy conservation equation is not needed in our model because temperature is assumed to be a constant inside the chamber during the withdrawal process. A barotropic state law can be therefore established in this region. However, even for barotropic flows, the energy conservation equation remains coupled to the mechanical ones due to the Joule effect. If this contribution is not considered in the energy equation, the use of a barotropic state law will uncouple the thermal equation from the mechanical ones. Therefore only mass and momentum equations are required in our case. Using a spatial notation and Cartesian coordinates, they can be written as follows :

\section{Continuity equation (mass conservation)}

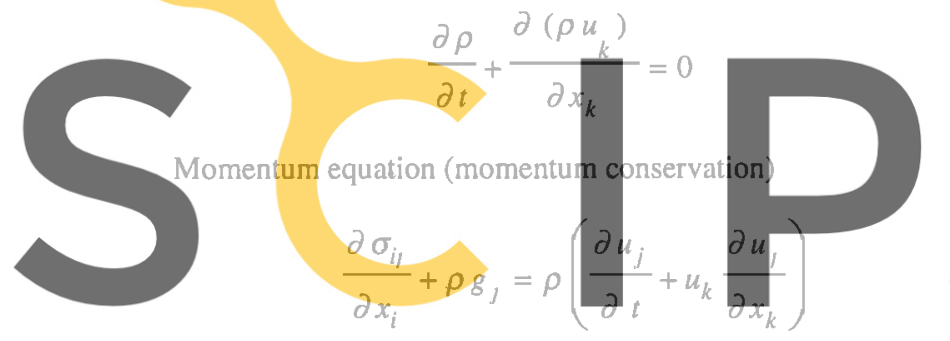

(2)

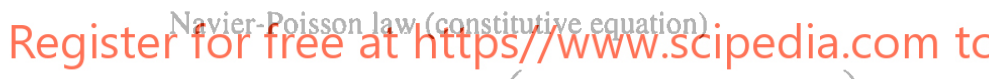

$$
\sigma_{i j}=-P \delta_{i j}+\mu\left(\frac{\partial u_{i}}{\partial x_{i}}+\frac{\partial u_{j}}{\partial x_{i}}-\frac{2}{3} \frac{\partial u_{k}}{\partial x_{k}} \delta_{i j}\right)
$$

Barotropic state law

$$
\rho=\rho(\mathrm{P})
$$

where (see notation section) $\rho$ is density, $\mu$ is viscosity, $P$ is pressure, $u_{t}$ is the ith component of the velocity vector, $\sigma_{l j}$ are the components of the Cauchy stress tensor, and $g_{j}$ is the jth component of the gravity. In all the equations of this paper, indices run from 1 to 2 or 3 (space dimensions) and repeated indices always imply summation. The proposed barotropic state law for magmas inside the chamber (4) is discussed in the section 2.3. Defining the viscous stress tensor $\tau$ (excluding pressure isotropic term in $\sigma$ ) as

$$
\tau_{i j}=\sigma_{i j}-\frac{1}{3} \sigma_{k k} \delta_{l j}=\sigma_{i j}+P \delta_{i j}
$$

the set of equations can be expressed in its pure conservative form

$$
\frac{\partial \vec{V}}{\partial t}+\frac{\partial \vec{C}_{i}}{\partial x_{i}}+\frac{\partial \vec{D}_{i}}{\partial x_{i}}+\vec{S}=0
$$

where the conservative variables (mass and momentum)

$$
\vec{V}^{T}=\left(\rho, \rho u_{1}, \rho u_{2}, \rho u_{3}\right)
$$

are transported by means of convection through the convective tluxes

$\bar{C}_{t}^{T}=\left(\rho u_{\imath}, \rho u_{\imath} u_{1}+\delta_{\imath 1} P, \rho u_{i} u_{2}+\delta_{\imath 2} P, \rho u_{\imath} u_{3}+\delta_{\imath 3} P\right)$

and by means of diffusion through the diffusive fluxes

$$
\vec{D}_{i}^{T}=\left(0,-\tau_{t 1},-\tau_{i 2},-\tau_{i 3}\right)
$$

The source term is

$$
\vec{S}^{T}=-\left(0, \rho g_{1}, \rho g_{2}, \rho g_{3}\right)
$$

where $g$, is the ith component of the gravity acceleration vector. Then, in this conservative form, the equations to solve are

\section{Continuity equation}

$$
\frac{\partial p}{\partial t}+\frac{\partial}{\partial x_{i}}\left(U_{i}\right)=0
$$

Momentum equation

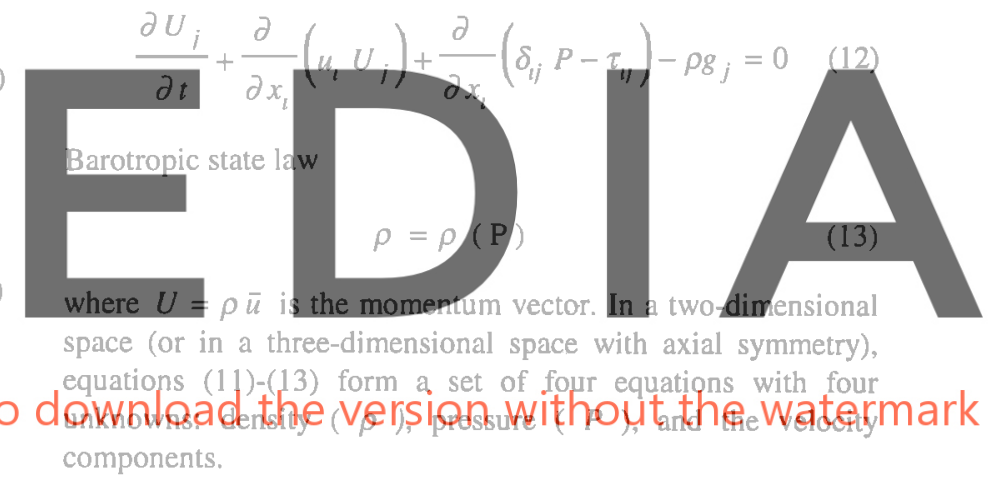

\subsection{The State Law}

In order to close the problem, a state law (4) is required. For high-viscosity magmas above the exsolution level, the level where exsolution due to melt oversaturation begins, and below the fragmentation level, where the flow is characterized by a mixture of gas with pyroclasts and lithics, the mixture can be considered as a bubbly flow, characterized by a liquid continuum with dispersed gas bubbles and crystals in thermal and mechanical equilibrium [Papale, 1996]. In this bubbly flow regime, the pressure difference between both phases (liquid and the gas bubbles within) can be ignored [Sparks, 1978]. The gas bubbles and the liquid are in mechanical equilibrium and can be considered to move at the same velocity along the conduit because buoyancy effects are negligible compared to viscous drag, even for centimetric bubble sizes (notwithstanding this, the formation of large bubbles in high-viscosity magmas is unlikely because growth by coalescence is impeded). We aim to find a state law for the mixture inside the magma chamber and in the lowermost part of the conduit, that is, in the bubbly flow regime domain, where the mixture is a continous medium with density:

$$
\rho=\alpha \rho_{g}+(1-\alpha) \rho_{s} \cong \alpha \rho_{g}+(1-\alpha) \rho_{l}
$$


with $\alpha$ being the gas volume fraction, $\rho_{g}$ being the gas density, $\rho$, being the density of the melt (containing dissolved volatiles) and $\rho_{l}$ being the liquid density. In (14) it is assumed that the dissolved volatiles do not change the bulk density of the liquid. This homogeneous approximation for the mixture is commonly assumed in the literature [Wilson et al., 1980; Giberti and Wilson, 1990], at least in the lowermost part of the conduit and for high-viscosity magmas. The gas phase is assumed to behave as a perfect gas [Vergniolle and Jaupart, 1986], with

$$
P=\rho_{g} Q T
$$

where $T$ is temperature, $\rho_{g}$ is the gas density, and $Q$ is a gas constant ( $Q=461.66 \mathrm{~J} /{ }^{\circ} \mathrm{K} \mathrm{kg}$ for water). For the liquid component, a linearized expression in terms of the compressibility modulus $\beta$ can be assumed [Blake, 1984]:

$$
\rho_{l}=\rho_{0}\left(1+\beta\left(P-P_{0}\right)\right)
$$

where $\rho_{0}$ is the density at the pressure reference $P_{0}$. In order to find the state law, we should consider that the volatile species can be either dissolved in the melt or exsolved as a gas phase if the magma is above the exsolution level. Therefore a solubility law is also needed to determine the mass fraction of volatiles dissolved in the melt $\chi$ at given thermodynamic conditions. The simplest relationship under magmatic conditions is a Henry's law equation, where the solubility of the silicate melt depends

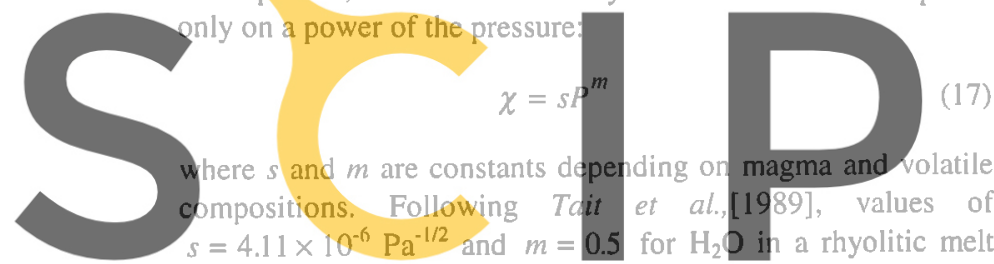

can be assumed. Using this solubility law, the critical pressure $P_{c}$

$$
P_{c}=\left(\frac{W \%}{s}\right)^{\frac{1}{m}}
$$

below which gas exsolution takes place depends only on the volatile content $(W \%)$. For simplicity, we assume that the exsolution occurs instantaneously if pressure descends below this critical value, and no energetic contributions are considered during the vesiculation process. Let $m_{d}$ be the mass of dissolved gas in the melt, $m_{l}$ be the mass of the liquid, $m_{g}$ be the mass of gas, $m_{\nu}$ be the mass of volatiles (dissolved plus exsolved, where $\left.m_{v}=m_{g}+m_{d l}\right), m_{s}$ be the mass of the melt (liquid plus dissolved gas, where $\left.m_{s}=m_{l}+m_{d l}\right)$, and $M$ be the total mass $\left(M=m_{l}+m_{v}=m_{1}+m_{h}\right)$. Then

$$
\chi=\frac{m_{d}}{m_{d}+m_{l}}=\frac{m_{d}}{m_{s}}
$$

is the gas mass fraction dissolved in the melt,

$$
W \%=\frac{m_{v}}{M}=\frac{m_{v}}{m_{v}+m_{l}}
$$

is the volatile mass fraction (dissolved plus exsolved),
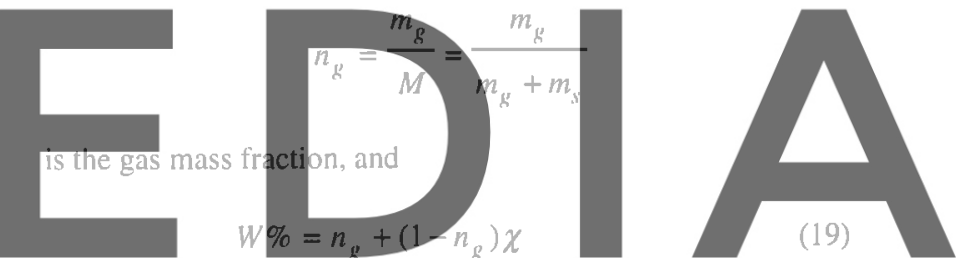

Register for free at https//Lww's,scipedia.com to download the version without the watermark

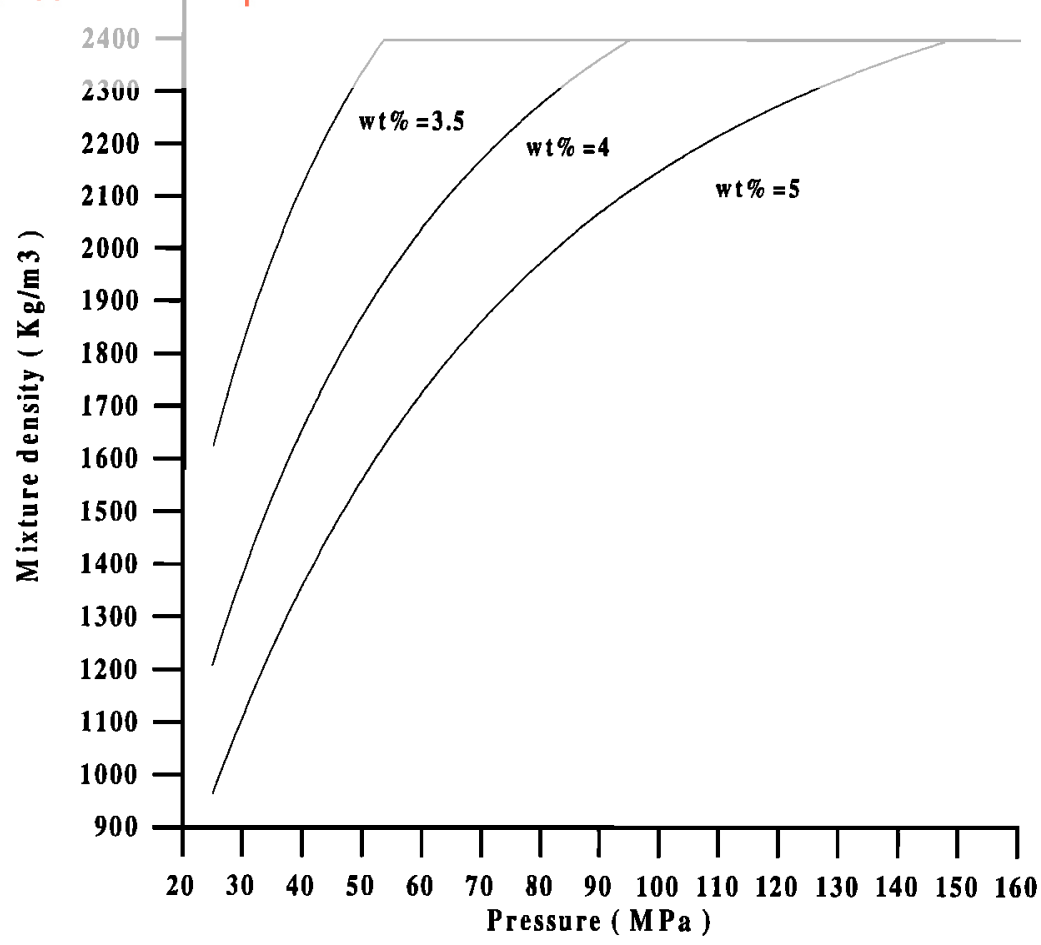

Figure 2. Magma density versus pressure given by the state law $\left(20^{\prime}\right)$. The results are for a rhyolitic magma with $T=850^{\circ} \mathrm{C}, \rho_{l}=2400 \mathrm{~kg} / \mathrm{m}^{3}$ and having water contents of $3.5,4.0$, and 5.0 in weight, respectively. Note how the exsolution level (the level where the mixture density becomes a constant equal to $\rho_{l}$ ) depends on the water content. 


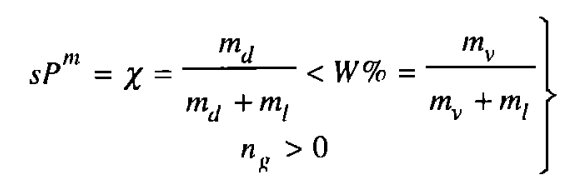

above exsolution level

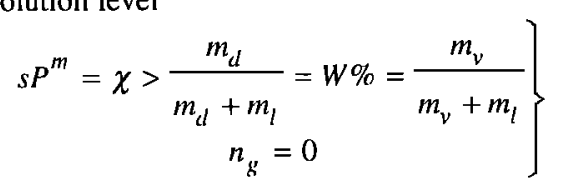

below exsolution level. Using equations (14) to (19) and after some rearrangement, one arrives at a final expression for the state law:

$$
\rho=\frac{\rho_{l}}{1+\left(\frac{\rho_{l} Q T}{P}\right)\left(\frac{W \%-s P^{m}}{1-s P^{m}}\right) l_{\theta}\left(P_{c}-P\right)}
$$

where $\theta$ is the step function (defined as $\theta=0$ for $P \geq P$, and $\theta=1$ for $P \leq P_{t}$ ). Note that $(20)$ is a general state law, not only applicable to vesiculated magmas. In general, the critical pressure $P_{c}$ at which gas exsolution begins will be attained inside the chamber depending on the chamber depth (lithostatic pressure) and on the volatile content. If this critical pressure is not attained, that is, if magma is not vesiculated, we have $\theta=0$ in (20), and the density is that of the liquid. In turn, liquid density , can be considered in genera depending also on the pressure, magma has values of the compress GPa [Touloukian et al., 1981], behaves as an incompressible flow the exsolution level. Hence liquid density $\rho_{l}$ is set as a constant in all computations, and the state law (20) becomes

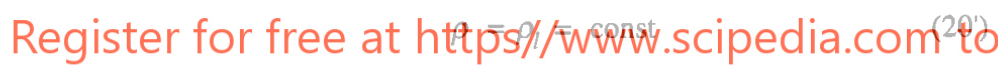
below the exsolution level

$$
\rho=\frac{\rho_{l}}{1+\left(\frac{\rho_{l} Q T}{P}\right)\left(\frac{W \%-s P^{m}}{1-s P^{m}}\right)}
$$

above the exsolution level.

Figure 2 shows the density profile of the magma predicted by the equation $\left(20 . b^{\prime}\right)$ for a rhyolitic magma with $\rho_{l}=2400 \mathrm{~kg} / \mathrm{m}^{3}$ at $850^{\circ} \mathrm{C}$. Note how the magma density is constant below the exsolution level and descends progressivelly above this level due to the volatile exsolution induced by the pressure decrease. Equation (20') has been also recently proposed by Bower and Woods [1997] as a suitable state law for the magma-volatile mixture.

\section{Numerical Method}

\subsection{Procedure}

The set of equations (11), (12), and (20) are numerically solved using a finite element method (FEM). The characteristics of the method are briefly summarized here. A full description of the algorithm is given by Codina et al. [1998]. Let us write the conservative equations (11) and (12) as

$$
\frac{\partial \rho}{\partial t}+\frac{\partial\left(\rho u_{k}\right)}{\partial x_{k}}=0
$$

and

$$
\frac{\partial U_{i}}{\partial t}=M_{i}-\frac{\partial p}{\partial x_{t}} \equiv R_{i}
$$

where $R_{1}$ is the ith component of the spatial operator and the body force and

$$
M_{i} \equiv-\frac{\partial}{\partial x_{1}}\left(\rho u_{l} u_{1}-\tau_{l}\right)+\rho g_{l}
$$

First, transport equations are time discretized using a traditional finite differences scheme. Second, the space discretization is done using a Galerkin FEM method. The momentum equation has a convective contribution appearing in (23) that could lead to numerical oscillations when discretizing the space using a standard Galerkin formulation. In this algorithm, the equations with convective terms are time discretized along the characteristics, thus providing a consistent artificial diffusion when the spatial discretization is done. This leads to the following time-discretized equations:

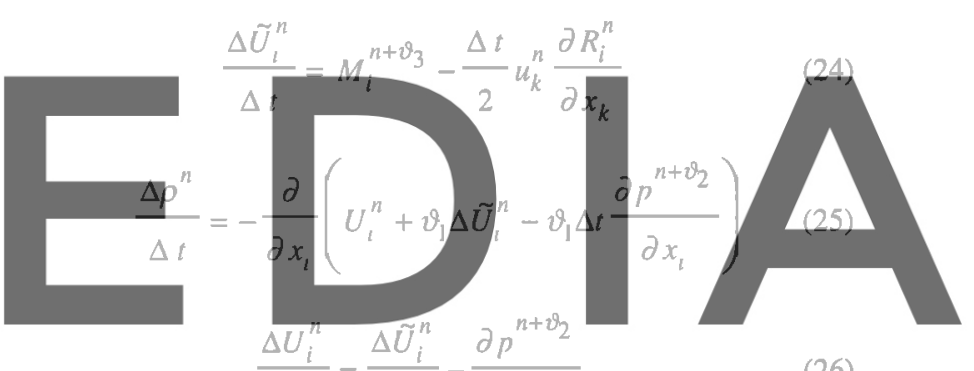

\section{download the versiton without the watermark}

where $\Delta t$ is the time step size, the superscripts denote time step level, and $\theta_{1}, \theta_{2}$, and $\theta_{3} \in[0,1]$. Equations (24) and (26) come frome the splitting of the momentum equation (discretized in time along the characteristics). We use a fractional step method thus introducing a new unknown to the problem, the fractional momentum $\widetilde{U}$. This technique was first proposed by Chorin [1967] and Temam [1969] for incompressible flows and allows the use of equal interpolation spaces for pressure and velocity fields. The splitting of the linear momentum equation produces a stabilizing effect on the pressure, eliminating the need for compatibility conditions, the Babuska-Brezzi conditions, when the incompressible limit is reached. This is an important point: in our model magma behaves either as a compressible flow or as a incompressible flow depending on pressure, so that we need an algorithm able to solve for both kind of flows. Once time discretization is done, the weak form of the equations is obtained, that is, time-discretized equations are projected into the usual space of test functions and integrated over the domain $\Omega$. Integrals with spatial secondorder derivates are integrated by parts. Finally, we can discretize the space using a standard Galerkin method where the test functions are equal to the shape functions. In order to stabilize the numerical scheme at high Reynolds number (convection dominated flows), the time derivatives are discretized along the characteristics [Codina et al., 1998], thus introducing the required artificial diffusion. This allows one to deal with high Reynolds number flows. Once the spatial disretization has been 
performed, the equations can be written in matrix form:

$$
\begin{gathered}
\mathbf{M} \frac{\Delta \overrightarrow{\tilde{\mathbf{U}}}^{n}}{\Delta t}=\overrightarrow{\mathbf{F}}_{\mathbf{1}}-\mathbf{K} \overrightarrow{\tilde{\mathbf{U}}}^{n+\vartheta_{3}} \\
\mathbf{M}_{\alpha} \frac{\Delta \overrightarrow{\mathbf{P}}^{n}}{\Delta t}+\vartheta_{1} \Delta t \mathbf{L} \overrightarrow{\mathbf{P}}^{n+\vartheta_{2}}=\overrightarrow{\mathbf{F}}_{2} \\
\mathbf{M} \frac{\Delta \overrightarrow{\mathbf{U}}^{n}}{\Delta t}=\mathbf{M} \frac{\Delta \overrightarrow{\tilde{\mathbf{U}}}^{n}}{\Delta t}-\mathbf{G} \overrightarrow{\mathbf{P}}^{n+\vartheta_{2}}+\overrightarrow{\mathbf{F}}_{\mathbf{3}}
\end{gathered}
$$

where $\mathbf{U}, \mathbf{P}$, and $\mathbf{U}$ are the vectors of nodal unknows and $\mathbf{M}, \mathbf{K}, \mathbf{G}$, and $\mathbf{L}$ are the standard mass matrix, the matrix coming from the viscous and convective terms in the fractional momentum equation, the matrix coming from the gradient operator, and the matrix coming from the Laplacian operator respectively. The matrix $\mathbf{M}_{\alpha}$ in the discrete version of the continuity equation (28) is defined as

$$
\mathbf{M}_{\alpha, i j} \equiv \int_{\Omega} \alpha N_{i} N_{j} d \Omega
$$

where $N$, is the shape function associated to the ith node of the FE mesh and $\alpha$ depends on the state law considered. Using the state law (20), one gets
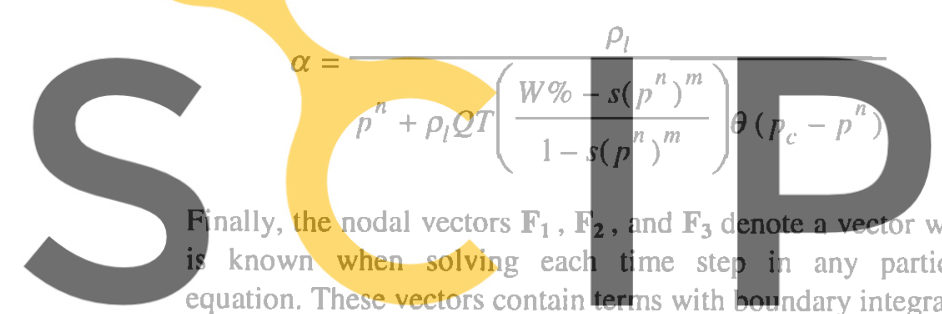
equation. These vectors contain terms with unum ary integrals in which some boundary conditions are prescribed. Each time step

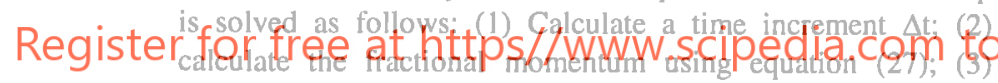

calculate the pressure using equation $(28) ;(4)$ calculate the density using the state law (20), and (5) calculate the momentum using equation (29). Some remarks concerning the implementation of the algorithm are required. Three parameters $\theta_{1}, \theta_{2}$, and $\theta_{3}$, with $\theta_{1} \in[0,1]$ have been introduced when discretizing in time the momentum equation $\left(\theta_{3}\right)$, the continuity equation $\left(\theta_{1}\right)$, and during the splitting $\left(\theta_{2}\right)$. Depending on its values the algorithm becomes explicit, semi-implicit or fully implicit. If $\theta_{2}=\theta_{3}=0$, the algorithm is explicit. In this case, the incompressible regime cannot be dealt with, since it can be shown that (28) becomes an identity when $\alpha=\rho_{l} / p$ in (31). This reflects the fact that pressure must be treated implicitly for incompressible flows. If $0<\theta_{2} \leq 1$ and $\theta_{3}=0$, the scheme is semi-implicit, and either pressure or density can be considered as unknows except in the incompressible limit. The semiimplicit scheme can be applied either in compressible flows or in incompressible flows and using the same interpolation functions for velocity and pressure (density) fields. In our case, the implicit scheme with the pressure as unknown is used for two reasons. First, because the state law (20) gives density as a function of pressure directly, while pressure as a function of density cannot easily be obtained, and second, because neither the boundary conditions at the outlet or the initial conditions are known in terms of density. The critical time step $\Delta t$ for a convection-diffusion equation can be computed as [Hirsch, 1990]

$$
\Delta t=\frac{F_{s}}{\frac{4 \varepsilon}{h^{2}}+\frac{|\vec{u}|}{h}}
$$

where $F_{\mathrm{s}}$ is a safety factor (less than 1 in the explicit scheme) and $h$ is the characteristic element dimension. For the continuity equation, $\varepsilon=0$, while for the momentum equation, $\varepsilon=\mu / \rho, \mu$ being the viscosity. If a stationary problem were considered, discrete time advance could be different for each equation and could be local or global. In our case, the time advance must be uniform because a transient problem is studied. Substituting typical magmatic values in (32), one gets $\Delta t \approx F, / 10$, so that the maximum time step able to ensure the stability of the algorithm is extremely small. Due to this limitation, we performed all simulations using the fully implicit option $\left(\theta_{l}=\theta_{3}=0\right)$, where $F_{3}$ can achive values up to 20 without any stability problem. Due to the small critical time step, one has to solve the whole set of equations to advance just 2 in real time. Thousands of time steps are required to simulate the entire withdrawal process. Examples of the reliability of the code are given in Zienkiewicz et al. [1995].

\subsection{Boundary and Initial Conditions}

Magma inside the chamber is always in a subsonic regime [Spera, 1984]. Under these circumstances, either pressufe of
density must be prescribed as a boundary condition at the
computational outlet [e.g., Hirsch, 1990]. The coupling betyeen
the modeling of nagma ascent, magma chamber processes, and
the mechanical problem of host rock behavior should be
considered because the processes which dccur in each of these
demains affect the dynamies of the others. When each of these domains (chamber, conduit, and host rock) is modeled as an

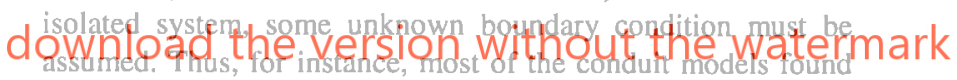

in the literature assume a pressure at the conduit entrance when in fact it is an unknown of the problem. In our computations, the outlet is set at $500 \mathrm{~m}$ above the entrance of the conduit, and therefore the computational outlet does not coincide with the physical one (the vent). We cut the computational domain in the middle of the conduit in order to ensure the validity of equation (20), that is, in order to keep the flow within the bubbly flow regime (see the appendix). Pressure at the computational outlet is prescribed to the lithostatic, despite the fact that conduit models predict a magma pressure drop below the lithostatic value inside the conduit [Papale and Dobran, 1993, 1994]. However, this assumption can be justified in gross terms because the average stress field within the conduit cannot be too far from lithostatic in order for it to remain open against compression of the country rocks. Another possibility would be to not cut the conduit and therefore to set the pressure at the vent. This would probably produce a more realistic boundary condition. However, as discussed in the appendix, the state law (20) would not be realistic physically because the assumption of homogeneity would not be valid above the fragmentation level.

Boundary and initial conditions considered in our problem are schematically illustrated in Figure 3 . The no-slip condition (velocity vector equal to zero) is imposed at the chamber walls $(u=v=0$ ), while only the horizontal component of the velocity is set to zero at the symmetry axis $(x=0)$. Initial values are assigned to the velocity field and pressure. Magma is 


\section{Boundary conditions}

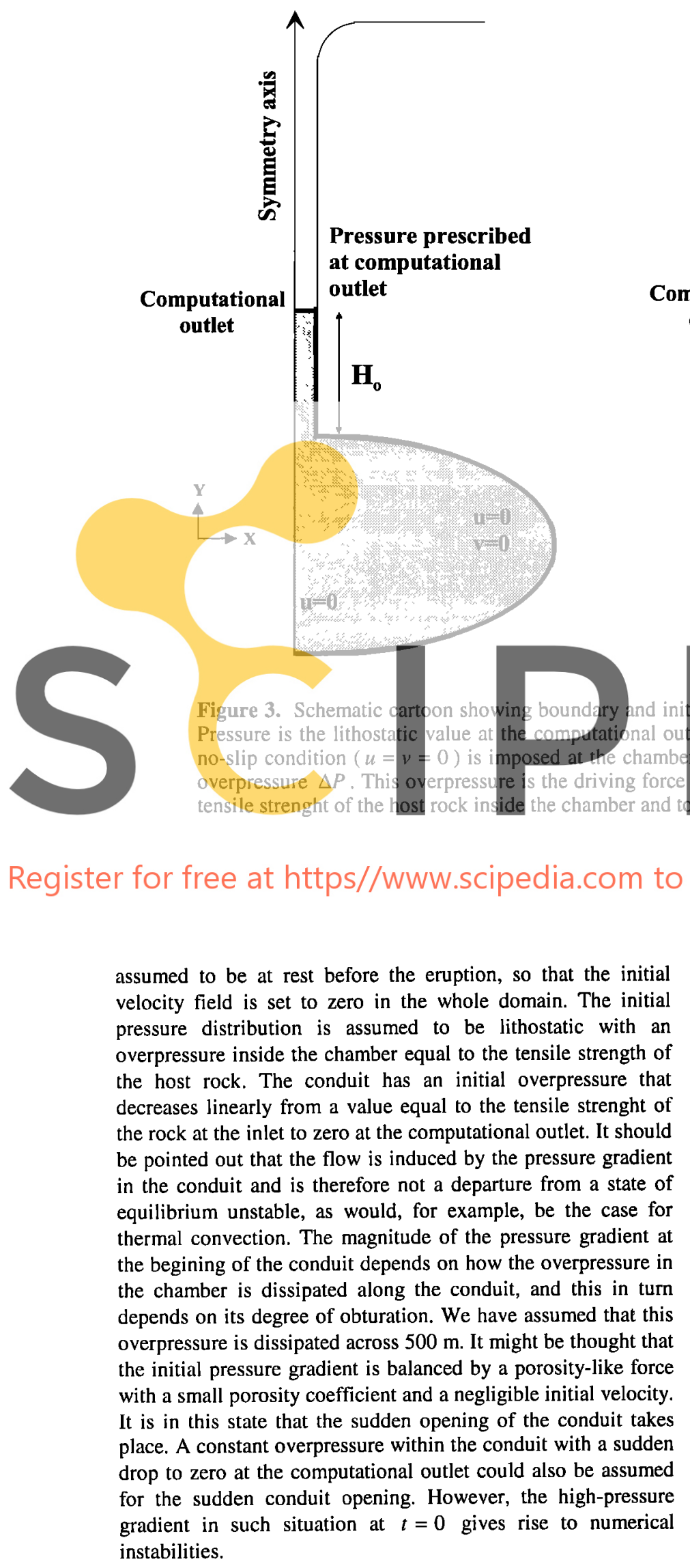

\section{Initial conditions}

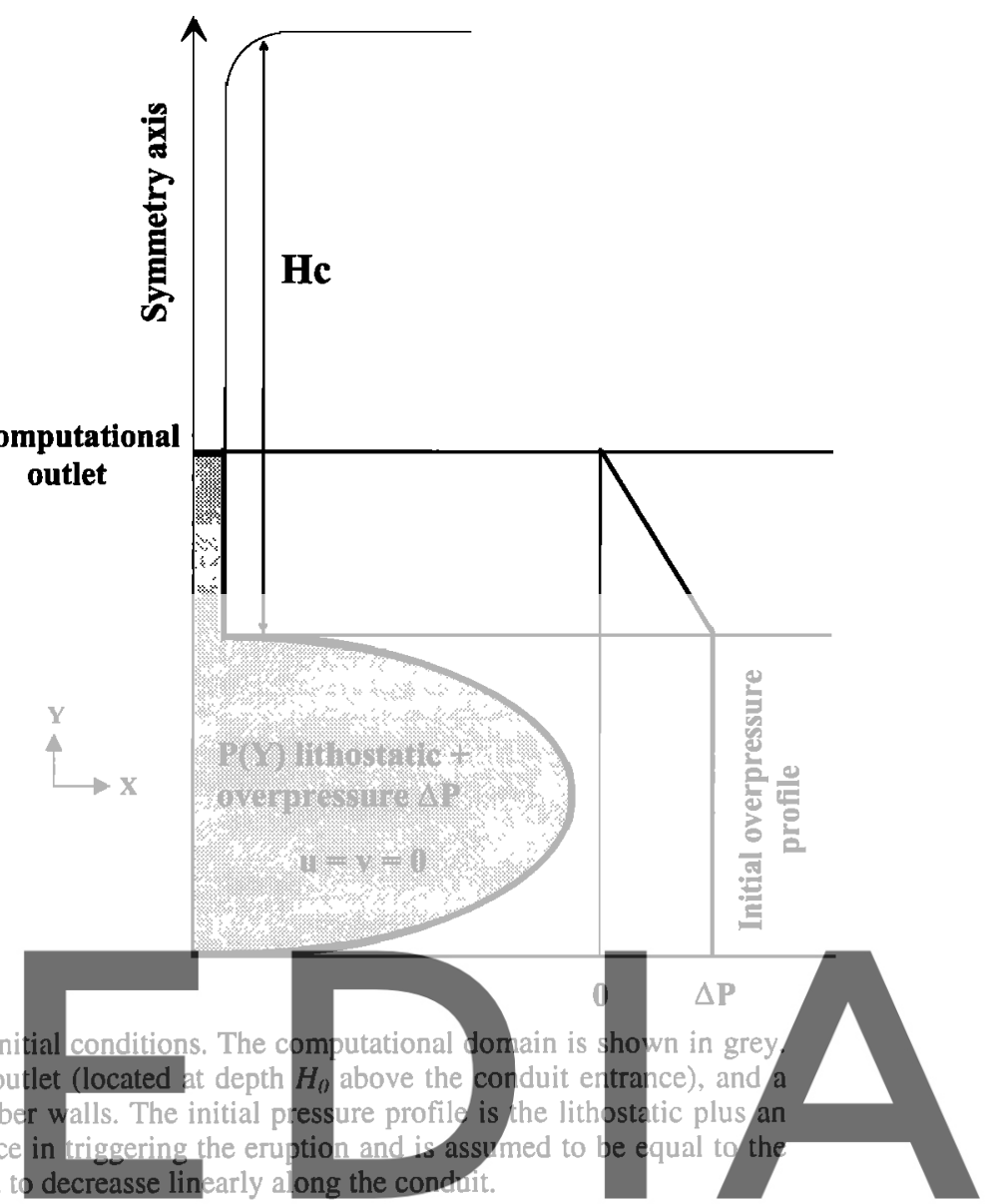




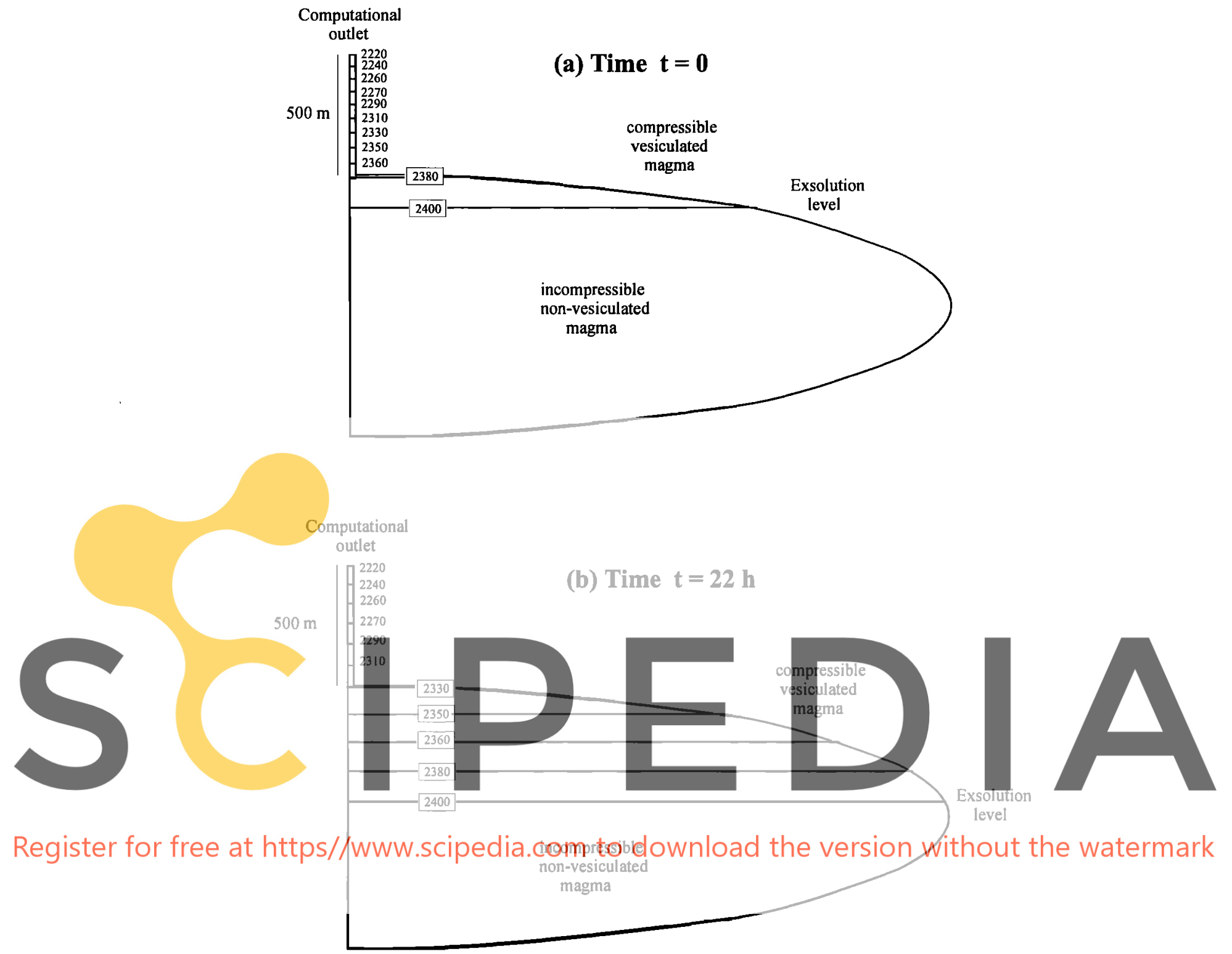

Figure 4. Density profiles for an ellipsoidal magma chamber with a major axis $a=2.5 \mathrm{~km}$, a minor axis $b=0.5 \mathrm{~km}$, a volume $V=4 / 3 \pi a^{2} b \cong 14 \mathrm{~km}^{3}$, and a conduit radius of $25 \mathrm{~m}$. The density is given in $\mathrm{kg} / \mathrm{m}^{3}$. Volatile content is set to 4.3 in wt\% and the lithostatic pressure at the computational outlet is $105 \mathrm{MPa}$. The nonvesiculated magma has a density $\rho=\rho_{l}=2400 \mathrm{~kg} / \mathrm{m}^{3}$ (a) Density profile at the onset of the eruption $(t=0)$ when only the uppermost part of the chamber is vesiculated. (b) Density profile after 22 hours of simulated eruption. Note the drop in the exsolution level due to the pressure decrease as the eruption proceeds.

\subsection{A General Overview}

In this section, the results for a particular numerical simulation are shown as an example of applicability of the model. Although the results presented are obtained by applying the model to a particular case, we stress those characteristics which are expected to be found in other simulations. A rhyolitic magma having $\rho_{l}=2400 \mathrm{~kg} / \mathrm{m}^{3}, T=850^{\circ} \mathrm{C}, \quad s=4.11 \times 10^{-6}$ $\mathrm{Pa}^{-1 / 2}, m=0.5$ and a water content of 4.3 in wt\% is assumed. The hypothetical shallow magma chamber is elliptical and has a major axis $a=2.5 \mathrm{~km}$, a minor axis $b=0.5 \mathrm{~km}$, a volume $V=4 / 3 \pi a^{2} b \cong 14 \mathrm{~km}^{3}$, and a conduit radius of $25 \mathrm{~m}$. The chamber has an initial pressure equal to the lithostatic pressure plus an overpressure $\Delta P=10 \mathrm{MPa}$. This overpressure is a typical value of the tensile strength of the host rocks [Touloukian et al.,
1981]. Note that this is an approximation because there is no straightforward relation between the overpressure within an ellipsoidal reservoir and the tensile stress induced in the elastic medium surrounding. The effect of the initial overpressure is discussed below. The lithostatic pressure at the computational outlet is set to $105 \mathrm{MPa}$. Note that we set the lithostatic pressure rather than the chamber depth $H_{c}$ in order to avoid assumptions about the host rock mean density. Thus, if the host rock density were $2500 \mathrm{~kg} / \mathrm{m}^{3}$, the computational outlet should be located at $4280 \mathrm{~m}$ below the Earth surface $\left(H_{c}=4780 \mathrm{~m}\right)$; if the host rock density were $2800 \mathrm{~kg} / \mathrm{m}^{3}$, the computational outlet should be located at $3820 \mathrm{~m}$ below the Earth surface $\left(H_{c}=4320 \mathrm{~m}\right)$.

All the numerical experiments yield similar results: the initial overpressure decreases exponentially as the eruption proceeds until it becomes zero and the eruption ceases. This pressure 


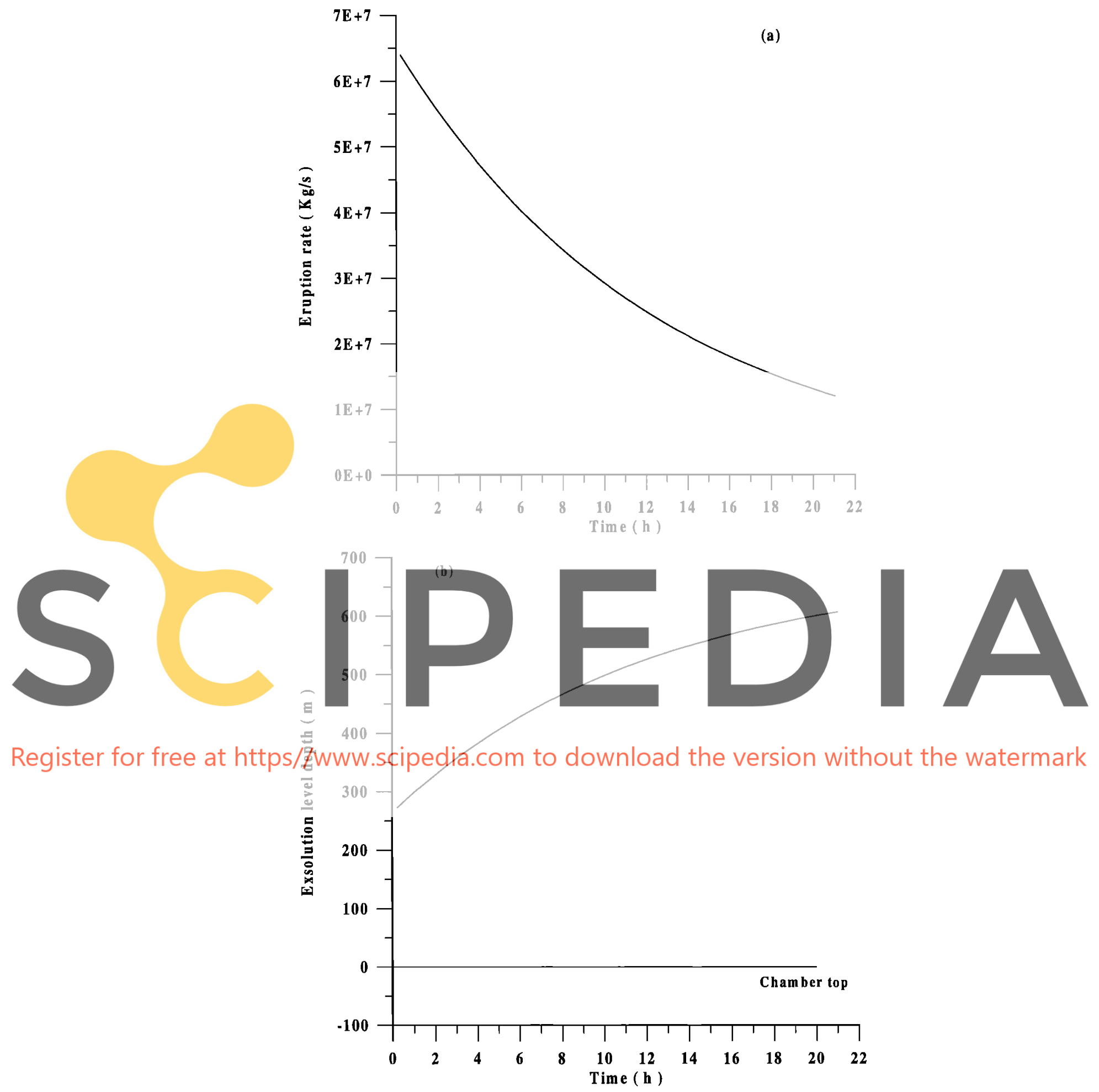

Figure 5. Results using the same chamber geometry and magma properties as in Figure 4. (a) Eruption rate as a function of time. (b) Position of the exsolution level as the eruption proceeds. The origin of the vertical axis is set at the top of the chamber ( $4 \mathrm{~km}$ below the surface) with the positive direction being downward.

decrease produces a drop in the exsolution level so that deeper parts of the chamber become progressively volatile oversaturated as the eruption proceeds. The upper oversaturated magma layer thus becomes progressivelly thicker and less dense. Figure 4 shows the density distribution for this elliptical magma chamber at the onset of the eruption and after 22 hours, when the initial overpressure has decreased by an order of magnitude.
Note that due to the pressure variations the position of the exsolution level descends hundreds of meters as the eruption proceeds. Figure 5 shows the eruption rate and the position of the exsolution level versus time for this particular simulation. A common characteristic of all the experiments is that the eruption rate reaches a peak inmediatelly after the onset of the eruption and then decreases exponentially as the eruption proceeds. 


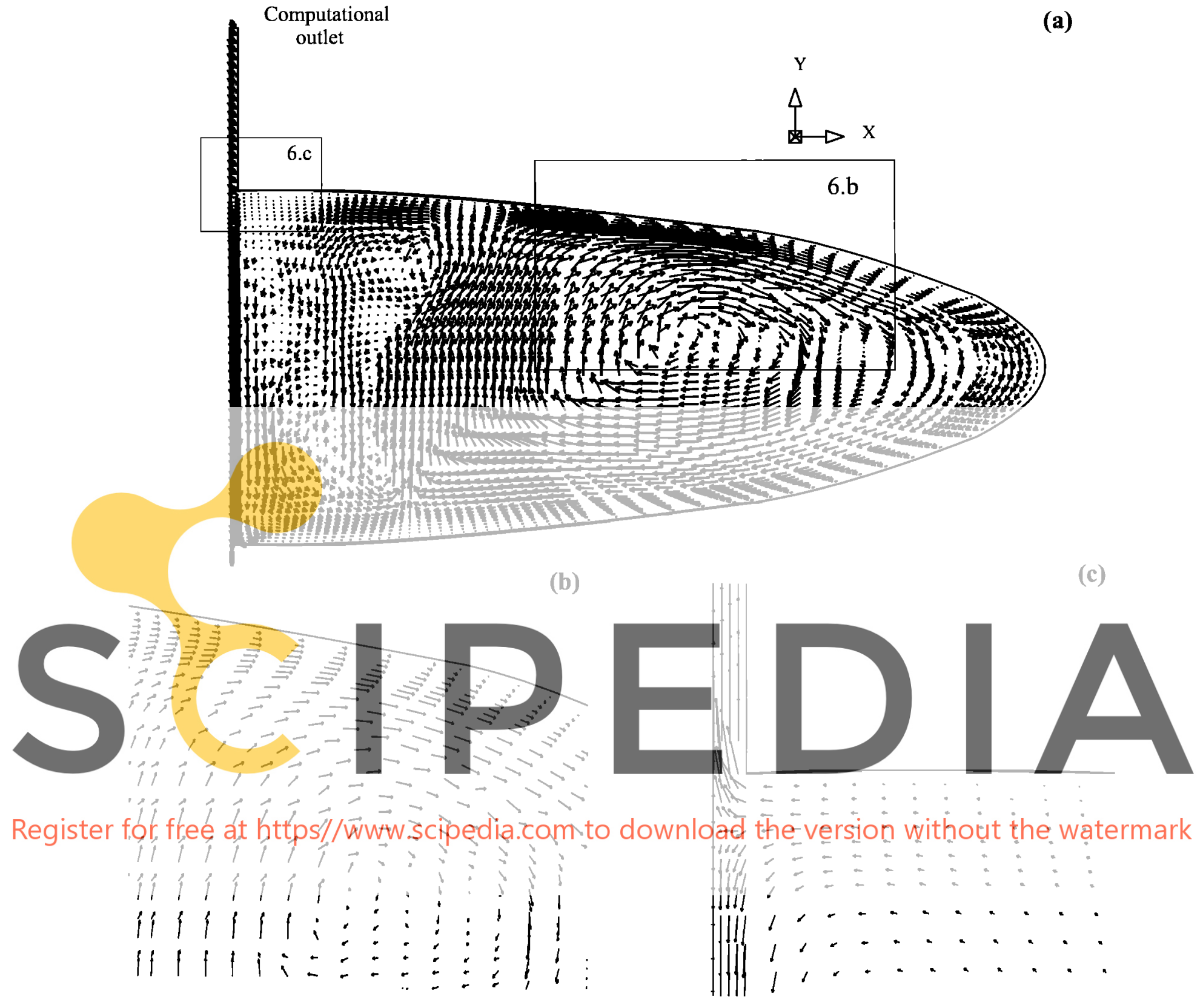

(a)

Figure 6. Velocity field inside the chamber after 22 hours of simulated eruption when using the same chamber geometry and magma properties as in Figures 4 and 5 . Although the velocity field is unsteady, its temporal variations are slow so that trajectories approach streamlines. Thus Figure $6 \mathrm{c}$ ilustrates how a parcel of magma reaches the conduit entrance laterally and is then either erupted or reinjected back into the chamber.

Nevertheless, it should be said that many Plinian eruptions present phases in which the eruption rate descends progressively only to then reach a new peak of intensity. These Plinian phases are commonly associated with tapping effects [e.g., Dobran, 1992], where the magma that reaches the conduit entrance changes its properties (viscosity, gas content, etc.) as the eruption proceeds, as a consequence of preeruptive heterogeneities. As we have assumed a chemically homogeneous magma chamber composition, our model is not able to reproduce such oscillations.

Although we do not model any specific volcanic system, the numerical results obtained are in good agreement with values observed in natural systems with comparable characteristics. For instance, in this example, we obtain a total erupted mass of aproximately $3 \times 10^{12} \mathrm{~kg}$. This value is comparable with those found in explosive volcanic eruptions: $0.6 \times 10^{12} \mathrm{~kg}$ in the 1980 Mount St. Helens eruption [Scandone and Malone, 1985], $5-7 \times 10^{12} \mathrm{~kg}$ in the Nisyros eruption [Barberi et al., 1988] or $8.6 \times 10^{12} \mathrm{~kg}$ in the 79 A.D. Vesuvius eruption [Barberi et al. $1981]$.

Figure $5 b$ shows the depth of the exolution level versus time. As also illustrated in Figure 4, the position of the exsolution level descends as the chamber is depressurized. In this particular example, the exsolution level is located initially at $275 \mathrm{~m}$ below 


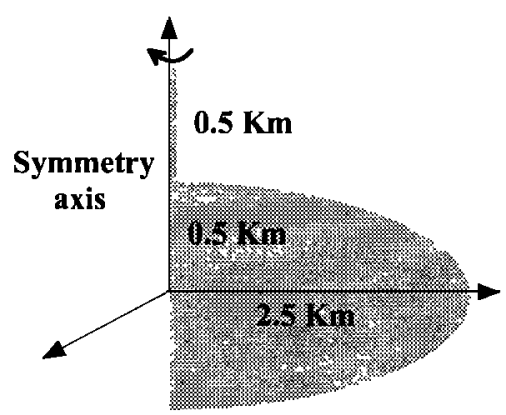

Chamber 1

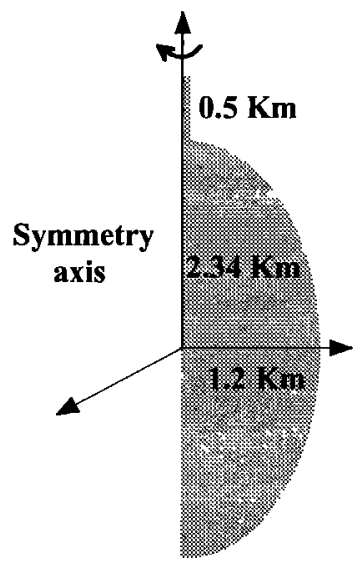

Chamber 3

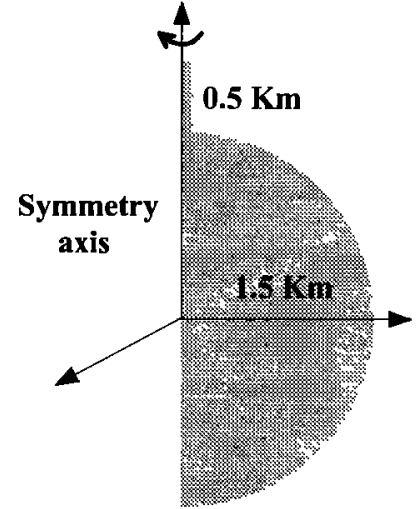

Chamber 2

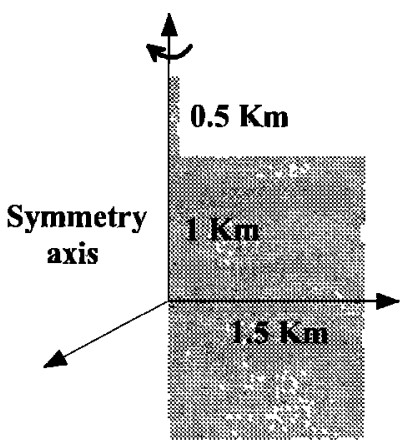

Chamber 4

Figure 7. Schematic cartoon showing the magma chamber geometries considered: chamber 1 is an oblate ellipsoid, chamber 2 is spherical, chamber 3 is a prolate ellipsoid, and chamber 4 is cylindrical. All the chambers have equal volume $V \cong 14 \mathrm{~km}^{3}$ and equal conduit radius $r=25 \mathrm{~m}$. The conduit has been cut $500 \mathrm{~m}$ above the chamber top to ensure the validity of $\left(20^{\prime}\right)$.

the conduit entrance and moves $300 \mathrm{~m}$ after 22 hours, reaching depths of up to $600 \mathrm{~m}$ below the chamber top. Figure 6 shows the velocity field inside the chamber after 22 hours of eruption. The magnitude of the velocity field is determined directly by the initial assumed overpressure. Convection could also be driven by thermal effects, although in the present simulation these have not been taken into account. To account for these effects, buoyancy forces would have had to be accounted for in the region where the flow is incompressible.

A common feature found during the withdrawal of a chemically homogeneous magma chamber is that the magma reaches the conduit entrance laterally as reflected in Figure 6c. Note that although the velocity field is unsteady, its variations are given in a characteristic timescale of hours. This means that we can safely approach the streamlines given by the trajectories of the particles. A volume of magma reaching this point is partly erupted through the conduit and partly reinjected into the chamber. Thus, a parcel of magma initially emplaced at the chamber top moves first laterally into the vicinity of the conduit entrance and is then either erupted or forced to descend before again moving up. As the parcel descends, exsolved volatiles within it are progressively redissolved back into the magma due to the pressure effect on the volatile solubility in the melt. This phenomenon has an important consequence: as we have assumed that magma behaves as an incompressible fluid below the exsolution level, any inflow into the undersaturated incompressible layer must produce an equal outflow in order to ensure the mass conservation. This movement is progressively transmited to the neighborhood of the parcels through viscous stresses, so that finally the whole magma chamber is affected by this induced movement, as reflected in Figure 6a. This is a common feature of all the numerical experiments, though its intensity and characteristics are obviously strongly dependent on magma viscosity and on chamber geometry. This phenomenon could have important consequences on the eruption products because magmas initially located at different depths could be simultaneously erupted. Trial et al. [1992] suggested that the presence of different compositions in the same Plinian diposits is not necesarily the consequence of a mixing process but may result from the withdrawal process itself when preeruptive gradients existed in the chamber. Our homogeneous flow model does not allow us to draw conclusions about stratified magma chambers with preeruptive density gradients but could explain some chemical heterogeneities, such as those involving trace element abundances, which have negligible effects on magma density. 
Table 1. Properties of the FEM Meshes

\begin{tabular}{lcccc}
\hline & Nodes & Elements & $\begin{array}{c}\text { Boundary } \\
\text { Elements }\end{array}$ & $\begin{array}{c}\text { Element } \\
\text { Type }\end{array}$ \\
\hline Chamber I & 3190 & 3080 & 218 & Q1 \\
Chamber 2 & 2680 & 2580 & 198 & Q 1 \\
Chamber 3 & 2680 & 2580 & 198 & Q1 \\
Chamber 4 & 2900 & 2775 & 248 & Q1 \\
\hline
\end{tabular}

All the elements are type Q1, that is, bilinear Lagrangian (four nodes per element).

\subsection{A Parametrical Study}

Many parameters such viscosity, gas content, magma chamber geometry or initial overpressure appear in the model, and its influences on the solution are not always clear. In this section, we aim to illustrate the influence of these parameters by showing how their values affect the results obtained in the numerical example previously described.

4.2.1. Chamber Geometry. In order to assess the importance of chamber geometry we consider four different magma chambers with volume $V \cong 14 \mathrm{~km}^{3}$ (Figure 7). Chamber 1 is oblate, chamber 2 spherical, chamber 3 prolate, and chamber 4 cylindrical. In all cases, the FEM meshes are structured and made of linear Lagrangian elements (four nodes per element). They properties are summarized in Table 1. The dimensions of these chambers are of the same order of magnitude as natural shallow magma chambers but are arbitrary in representing any particular volcanic system. The conduit has a radius of $25 \mathrm{~m}$ and again is terminated $500 \mathrm{~m}$ above the chamber because the state law $\left(20^{\prime}\right)$ may not be applicable in the uppermost parts of the conduit. Figure 8 shows the eruption rate at the conduit entrance versus time for this particular numerical experiment. An important result is that although shapes of the curves are similar in all four cases, the total mass erupted increases as the chamber becomes flatter and as the volumetric fraction found above the exsolution level increases. This can be explained by the fact that magma is nearly incompressible below the exsolution level (fully incompressible in our model) but that above this level, the mixture containing exsolved gas becomes much more compressible. Thus the total mass erupted corresponds only to the volume change in the upper compressible layer as a response to the pressure variation. In consequence, those chambers containing more magma in the upper oversaturated layer (like chambers 1 and 4 in our example) will erupt a greater mașs of material for a given pressure decrease. The total erupted mass is obtained by computing the area below the eruption rate curves versus time. In this example, we achive erupted fractions of the initial mass of $0.090,0.077,0.064$, and 0.078 for chambers 1 to 4, respectively. Recently, Bower and Woods [1997] have predicted that in saturated magma chambers, the chamber overpressure is only relieved when a fraction of 0.01-0.1 of the initial mass in the chamber has erupted. The numerical results are thus in excellent agreement with those predicted by the analytical model of Bower and Woods [1997]. Nevertheless, the use of a numerical model allows us to go one step further since we are able not only to compute the total mass erupted but also the temporal variation of the eruption rate and the influence of the chamber geometry.

4.2.2. Volatile Content. The influence of volatile content on the eruption rates and on the pressure variations is now considered. All the examples are obtained using magma chamber 1 (oblate ellipsoid), an initial overpressure of $\Delta P=10 \mathrm{MPa}$, a viscosity of $\mu=10^{5} \mathrm{~Pa} \mathrm{~s}$, and a density of $\rho_{l}=$ $2400 \mathrm{~kg} / \mathrm{m}^{3}$. The eruption rates obtained and the evolution of the exsolution level are shown in Figure 9. Although a small change in the content of volatiles does not change substantially the mass discharge rate, it has an important effect on the position of the

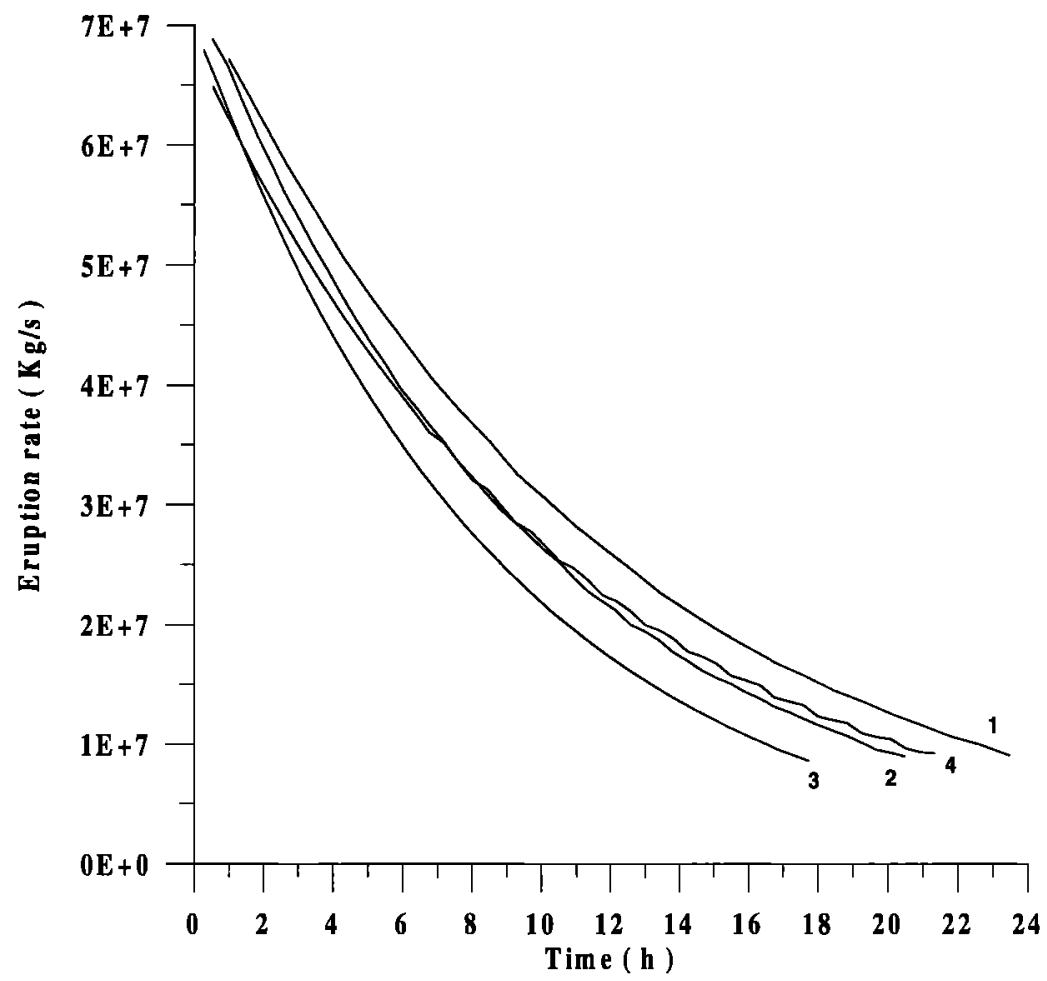

Figure 8. Eruption rate versus time for diferent magma chamber geometries. The chambers are those shown in Figure 7. 

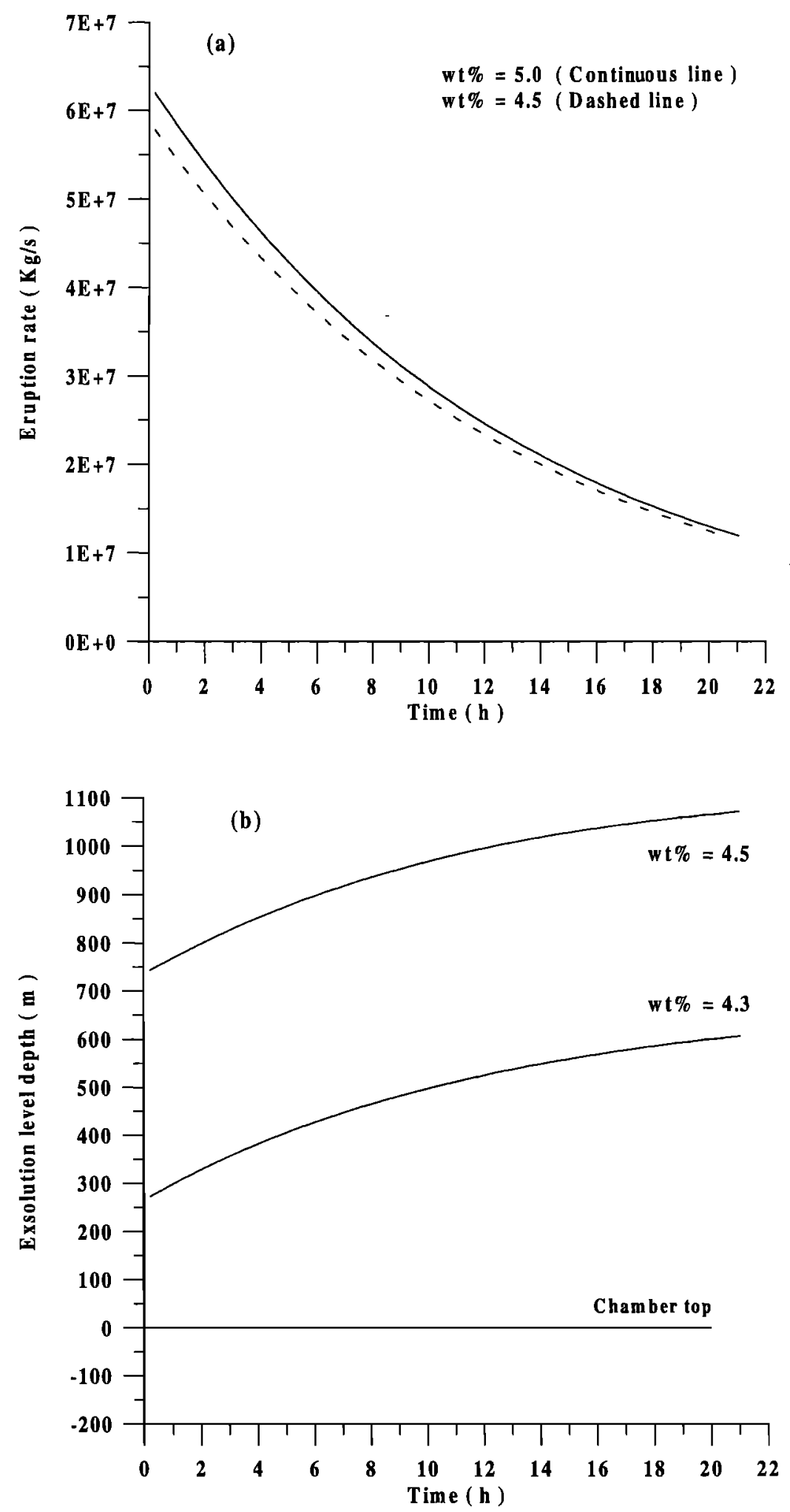

Figure 9. Results using chamber 1 , an initial overpressure of $\Delta P=10 \mathrm{MPa}$, a viscosity $\mu=10^{5} \mathrm{~Pa} \mathrm{~s}$, and a liquid density $\rho_{l}=2400 \mathrm{~kg} / \mathrm{m}^{3}$. (a) Eruption rate versus time for water contents of 4.5 and $5.0 \mathrm{in}$ wt $\%$. The exponential decrease is only weakly dependent on volatile content. (b) Position of the exsolution level for water contents of 4.3 and 4.5 in wt \%. The origin of the vertical axis is set at the top of the chamber. Note that the shape of the function does not vary substantially when the volatile content is changed, although the position of the exsolution level is dependent on the volatile content. 
exsolution level. A small increase in the volatile content produces a substantial deepening of the exsolution level. The total displacement is insensitive to the volatile content, but the initial and final positions are strongly dependent on its value.

4.2.3. Viscosity. Figure 10 shows the effect of varying viscosity on the magma overpressure, the position of the exsolution level, and the mass discharge rate when other parameters are kept constants. Figure 10 illustrates that the less viscous the magma, the faster the chamber becomes depressurized. The position of the exsolution level changes faster for less viscous magmas despite the fact that the initial and final positions are the same for a given pressure decrease ( $\Delta P=10 \mathrm{MPa}$ in this case). Although the total erupted mass is only weakly dependent on viscosity, this parameter plays a major role in controlling the duration of the eruption. The more viscous the magma, the longer the eruption and the lower the erupted mass peak, that is, chambers containing a less viscous magma are more rapidly withdrawn and have a greater intensity peak.

4.2.4. Initial Overpressure. The influence of the initial overpressure is now considered. All of the examples presented are for a chamber 1 geometry (Figure 7), with a viscosity of $\mu=10^{5} \mathrm{~Pa} \mathrm{~s}$, a density of $\rho_{l}=2400 \mathrm{~kg} / \mathrm{m}^{3}$ and a water content of 4.3 in wt \%, but with initial overpressures of 5,10 , and $15 \mathrm{MPa}$ respectivelly (Figure 11 ). The total drop in the exsolution level is strongly dependent on the initial overpressure, as is its initial position, while its final position is invariant. Obviously, an increase in the initial overpressure yields an increase in the displacement of the exsolution level. This leads to a greater erupted mass because a significant volume of magma changes from volatile-undersaturated (incompressible) to volatileoversaturated (compressible) during the withdrawal process.

\section{Conclusions}

We have developed an axisymmetrical numerical model in order to find the temporal evolution of pressure, the position of the exsolution level, the velocity field, and the eruption rate during the withdrawal from a shallow volatile-rich felsic magma chamber, where the overpressure is due to volatile oversaturation. In this model, magma behaves as an incompressible fluid below the exsolution level and as a compressible gas-liquid mixture above this level. In this domain, magma is treated as a homogeneous two-phase flow, and we propose a barotropic state law (20') as suitable for the mixture under magmatic conditions.

The resulting set of equations is solved numerically using a finite element method (FEM). We propose an algorithm able to deal simultaneously with compressible and incompressible flows. This uses a fractional step method which allows the use of equal interpolation spaces for pressure and velocity fields.

Figure 10. (a) The magma overpressure, (b) the position of the exsolution level, and (c) the mass discharge rate for two different viscosity values of $\mu=10^{4} \mathrm{~Pa} \mathrm{~s}$ and $\mu=10^{5} \mathrm{~Pa} \mathrm{~s}$, with the other parameters kept the same as in Figure 9. The less viscous the magma, the faster the chamber becomes depressurized and the faster is the descent of the exsolution level. Chambers containing less viscous magma are thus more rapidly withdrawn and have a greater peak intensity of erupted mass.
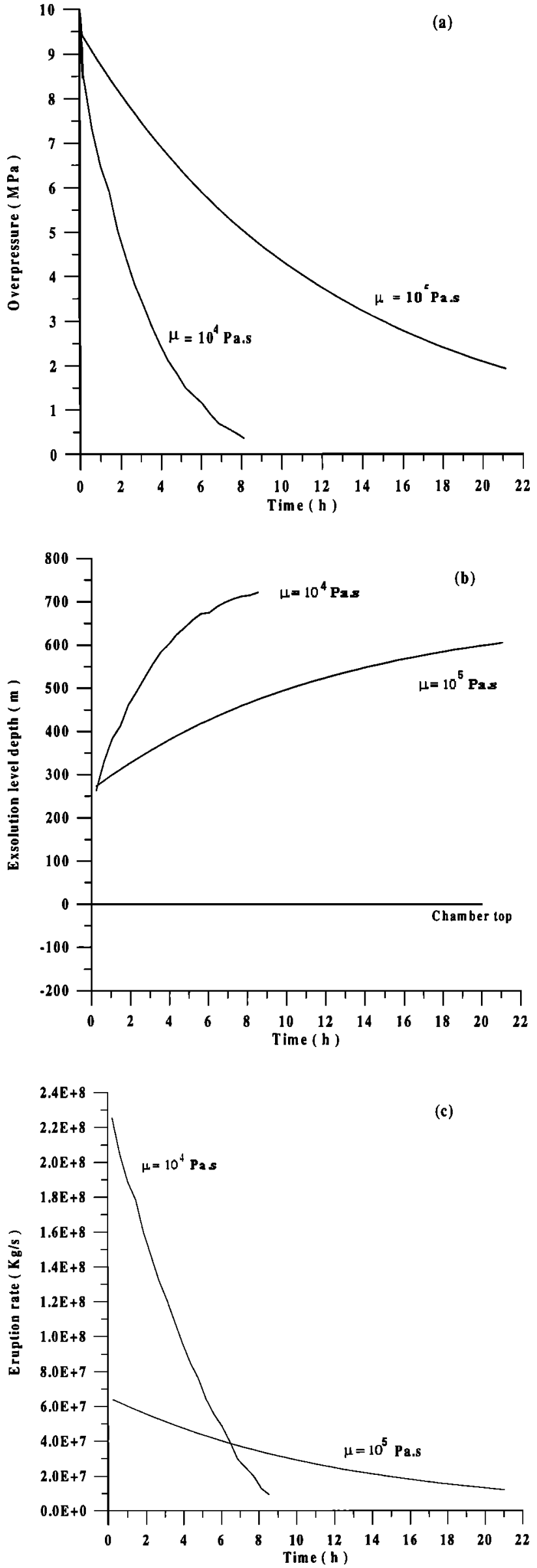

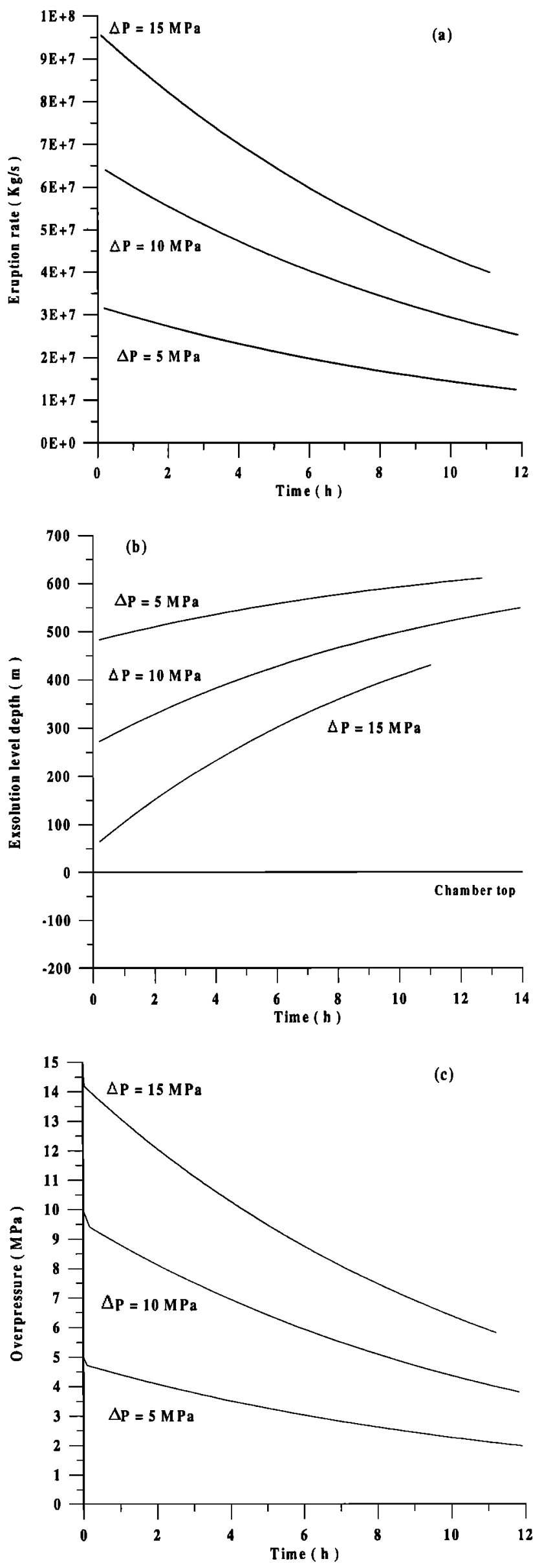

The artificial diffusion required in the equations with convective terms is obtained by discretizing in time along the characteristics. By assuming axial symmetry, we solve a threedimensional problem like a two-dimensional using cylindrical coordinates.

The results obtained show that the pressure at the conduit entrance decreases exponentially as the eruption proceeds. This pressure decrease produces a shifting of the exsolution level, so that deeper parts of the chamber become progressively volatile oversaturated. As previously proposed by Trial et al. [1992] for open magma chambers in which the trigger for the eruption is magma inflow into the chamber, our numerical experiments suggest that in closed, homogeneous magma chambers, magma from different depths can be erupted simultaneously. This may explain some chemical heterogeneities in the erupted products, such as different abundances of trace elements, which are not necessarily related to magma density. The total erupted mass predicted by the experiments is in good agreement with the results of Bowers and Woods [1997], who find that in closed volatile-saturated magma chambers, a mass fraction of 0.01-0.1 of the initial mass is erupted. In adition, the numerical simulations allow us to predict not only the total mass erupted but also its temporal variation for any chamber geometry and set of magma properties.

For chambers of equal volume and magma properties, the qualitative behavior is insensitive to the chamber geometry. However, the total erupted mass increases as the chamber becomes flatter because flat chambers have a major volumetric fraction of compressible magma. It should be pointed out that geometry would play a major role when considering the coupling with the mechanical problem. In natural systems, the stress field arround the chamber is directly related to its geometry. The stress field controls the opening, propagation and closure of those fractures that allow the magma to erupt. By assuming rigid walls, our model partially neglects the influence of geometry.

A small change in the magma volatile content does not substantially change the mass discharge rate but has an important influence on the position of the exsolution level. A small increase in volatile content produces a substantial deepening of the exsolution level. The exsolution level drops faster in less viscous magmas although the initial and final positions are insensitive to viscosity. The total erupted mass is weakly dependent on viscosity, although viscosity plays a major role in controling the duration and the peak intensity of the eruption. An increase in the initial overpressure, here associated with the tensile strength of the host rock, produces an increase in the total displacement of the exsolution level. As a consequence, when the initial overpresure is high we see a greater erupted mass because a major volumetric fraction of magma changes from volatile-undersaturated (incompressible)

Figure 11. (a) The mass discharge rate, (b) the position of the exsolution level, and (c) the overpressure evolution for different initial overpressures of 5,10 , and $15 \mathrm{MPa}$. An increase in the initial overpressure can be accounted for by an increase in the tensile strenght of the host rock. The total drop in the exsolution level depends strongly on the initial overpressure, although its final position is the same in all cases. Increasing the initial overpressure yields a greater erupted mass because a major volumetric fraction of magma changes from undersaturated to oversaturated during the depressurization process. 


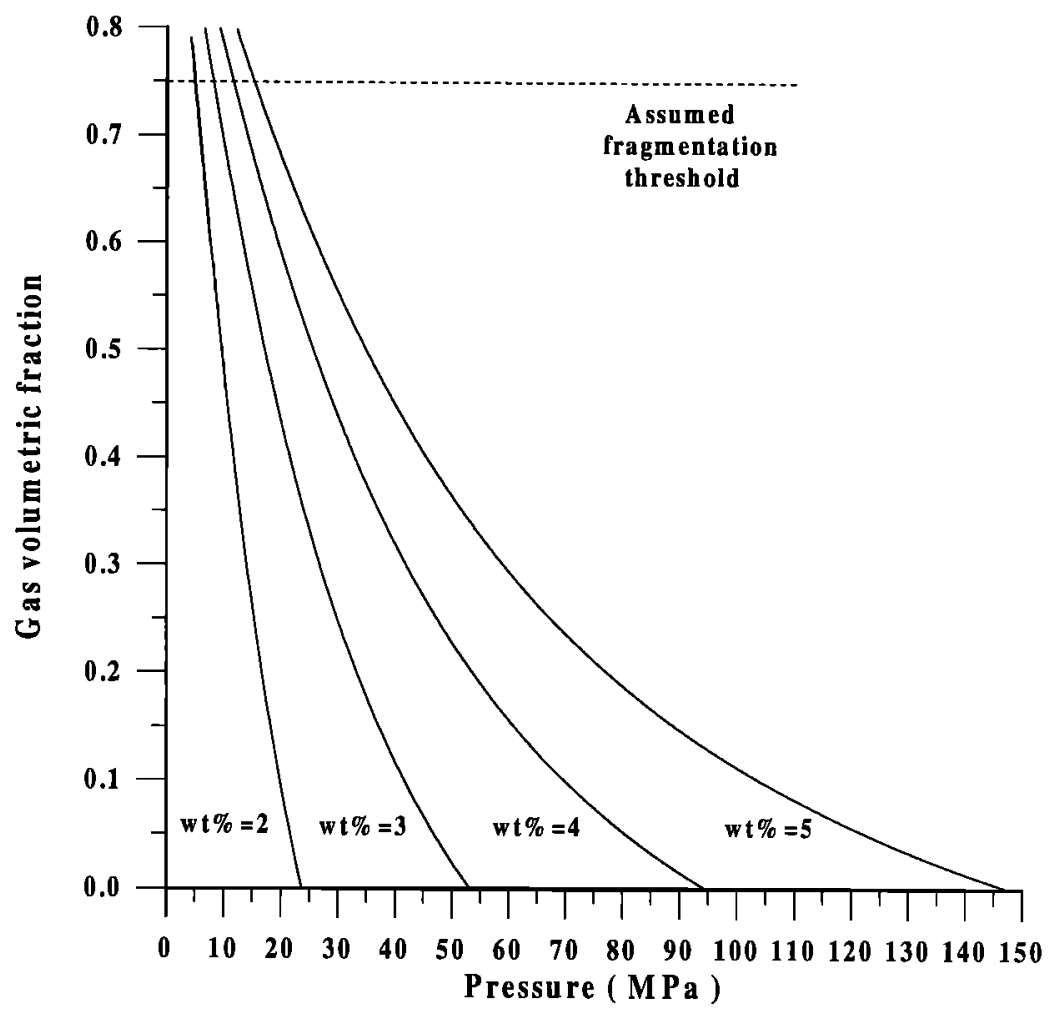

Figure 12. Gas volumetric fraction $\alpha$ predicted by the state law (20). Assuming a fragmentation threshold for $\alpha=0.75$ [e.g., Sparks, 1978], the domain in which the state law (20) is valid can be estimated quantitatively. Above the fragmentation level, at the uppermost part of the conduit, the bubbly flow regime cannot be maintained and the assumptions of the state law are physically unrealistic. Results for volatile contents of $2,3,4$, and 5 in wt \% respectively. The fragmentation level predicted by (20) is constrained to lie in the uppermost part of the conduit, even for high volatile contents.

to volatile-oversaturated (compressible) during the withdrawal process.

Our model constitutes a first attempt to numerically model the dynamics and temporal evolution of the most relevant physical parameters during magma withdrawal from a closed chamber. The relative importance of convective flow induced by overpressure and convective flow induced by thermal convection requires further study. Future models should also take account of chemical inhomogeneities in the magma, as well as the coupling with the mechanical problem. If a constitutive model for the host rock were coupled with this model, it should be possible to determine under which conditions of magma composition, chamber geometry, chamber depth, stress field, etc., a Plinian central vent eruption could generate a ring fault system and become a caldera-forming eruption.

\section{Appendix. Discussion on Boundary and Initial Conditions}

The barotropic state law (20) has been deduced assuming that the gas bubbles and the liquid are in mechanical equilibrium. As magma flows through the conduit, the gas bubbles grow by diffusion and by decompression. Growth by coalescence is inhibited in silicic magmas because gas bubbles are unable to come in contact by desplacing surrounding high-viscosity magma. The volumetric fraction of gas progressively increases as magma ascends and eventually reaches a critical bubble packing leading to magma fragmentation. Above this fragmentation level, the bubbly regime cannot be sustained, assumptions of mechanical equilibrium are no longer valid, and the state law (20) becomes physically unrealistic. The gas volume fraction $\alpha$ can be computed combining (14), (15), and (20). The result is shown in Figure 12, and reflects the need to limit the computational domain in order to ensure the validity of equation (20). We set the computational outlet at $500 \mathrm{~m}$ above the conduit entrance. This is an arbitrary choice but sufficient to ensure the validity of the state law even for very shallow magma chambers.

\section{Notation}

We use $f^{n+\theta} \equiv \theta f^{n+l}+(I-\theta) f^{n}$ and $\Delta f^{n} \equiv f^{n+I}-f^{n}$ for any function $f$. The superscript denotes time step level. Bold vectors stand for vectors of nodal unknowns, and $\mathbf{F}_{\mathbf{i}}$ denotes a vector which is known at the moment of solving a particular equation.

a semimajor axis (ellipsoidal chamber).

$b \quad$ semiminor axis (ellipsoidal chamber).

$\overline{\mathbf{g}}$ gravity vector.

$F_{\text {、 }}$ safety factor.

$H_{0}$ computational conduit height.

$H$, magma chamber depth.

$m$ solubility law exponent.

$m_{d}$ mass of dissolved gas.

$m_{g} \quad$ mass of exsolved gas.

$m_{l} \quad$ mass of liquid magma. 
$m$, mass of the melt

$m_{v} \quad$ mass of volatiles.

$M$ total mass.

$n$ molecular mass $\left(18 \mathrm{~kg} / \mathrm{kmol}\right.$ for $\left.\mathrm{H}_{2} \mathrm{O}\right)$.

$n_{h^{\prime}} \quad$ exsolved gas mass fraction.

$P$ pressure.

$P_{\text {c }} \quad$ critical (exsolution) pressure.

$Q$ gas constant.

$r \quad$ conduit radius.

$R \quad$ universal gas constant $\left(8314 \mathrm{~J} /{ }^{\circ} \mathrm{K} \mathrm{kmol}\right)$

$s \quad$ solubility law constant.

$T$ temperature.

$\vec{u} \quad$ velocity vector.

$u \quad x$ velocity component.

$\vec{U} \quad$ momentum vector

$\tilde{U}$ fractional momentum vector.

$v \quad y$ velocity component.

W\% volatile mass fraction.

$\alpha \quad$ gas volumetric fraction.

$\beta \quad$ compressibility modulus.

$\Delta P$ magma overpressure.

$\chi \quad$ solubility law ; maximum allowable mass fraction of gas into solution

$\mu \quad$ viscosity.

$\rho$ mixture density.

$\rho_{g} \quad$ gas density.

$\rho_{l} \quad$ liquid density.

$\rho_{s} \quad$ melt (liquid plus exsolved gas within) density.

$\sigma_{i j} \quad$ Cauchy stress tensor components.

$\tau_{i j} \quad$ viscous stress tensor components.

$\boldsymbol{\theta}$ step function.

$\theta_{t} \quad$ parameters of the algorithm.

Acknowledgments. This research has been founded by the European Commission contract ENV4-CT96-0259 and the CICYT project AMB96-0498-C04. A. Folch is grateful for a CIRIT research fellowship. We thank $M$. Bonafede and $M$. Ogawa for their constructive reviews and G.J. Ablay for improving the English text.

\section{References}

Barberi, F., H. Bizourard, R. Clocchiatti, N. Metrich, R. Santacroce, and A. Sbrana, The Somma-Vesuvius magma chamber: A petrological and volcanological approach, Bull. Volcanol., 44, 295-315, 1981.

Barberi, F., J.M. Navarro, M. Rosi, R. Santacroce, and A. Sbrana, Explosive interactions of magma with ground water: Insights from xenolits and geothermal drillings, Rend. Soc. Ital. Mineral. Petrol., 43, $901-926,1988$.

Blake, S., Volcanism and dynamics of open magma chambers, Nature, 289, 783-785, 1981.

Blake, S., Volatile oversaturation during the evolution of silicic magma chambers as an eruption trigger, J. Geophys. Res., 89, 8237-8244, 1984.

Bower, S., and A. Woods, Control of magma volatile content and chamber depth on the mass erupted during explosive volcanic eruptions, $J$. Geophys. Res., I02, 10273-10290, 1997.

Buresti, G., and C. Casarosa, One-dimensional adiabatic flow of equilibrium gas-particle mixtures in long vertical ducts with friction, $J$. Fluid Mech., 203, 251-272, 1989.

Chorin, A.J., A numerical method for solving incompressible viscous problems, J. Comput. Phys., 2, 12-36, 1967.
Codına, R., M.Vázquez, and O.C. Zienkiewicz, A general algorithm for compressible and incompressible flow -Part III. The semi-implicit form, Int. J. Numer. Methods Fluids, 27, 13-32, 1998.

Dobran, F, Nonequilibrium flow in volcanıc conduits and application to the eruptions of Mt St. Helens on May 18, 1980, and Vesuvius in AD 79. J. Volcanol. Geotherm. Res., 49, 285-311, 1992.

Giberti, G., and L. Wilson, The influence of geometry on the ascent of magma in open fissures, Bull. Volcanol, 52, 515-521, 1990.

Hirsch, C., Numerical Computation of Internal and External Flows, vol. 2, John Wiley, New York, 1990.

Huppert, H., and R.S.J Sparks, Double-diffusive convection due to crystalization in magmas, Annu. Rev. Earth Planet. Sci., 12, 11-37, 1984

Papale, P. Modeling of magma ascent along volcanic conduits: A review in The mitigation of volcantc huzurds, edited by $\mathrm{F}$. Barberi and $\mathrm{R}$. Casale, pp. 3-40, Office for Official Publications of the European Communities, Luxembourg, 1996.

Papale, P., and F Dobran, Modeling of the ascent of magma durng the plinian eruption of Vesuvius in Ad 79, J. Volcanol. Geotherm. Res., 58, 101-132, 1993.

Papale, P., and F. Dobran, Magma flow along the volcanic conduit during the Plinian and pyroclastic flow phases of the May 18, 1980, Mount St. Helens eruption, J .Geophys. Res., 99, 4355-4373, 1994.

Scandone, R., and S.D. Malone, Magma supply, magma discharge and readjustment of the feeding system of the Mount St.Helens during 1980, J. Volcanol. Geotherm. Res., 23, 239-262, 1985.

Smith, R.L., Ash flow magmatısm, Spec. Pap. Geol. Soc. Am., 180, 5-27, 1979.

Sparks, R.S.J., The dynamics of bubble formation and growth in magmas: A review and analysis, J. Volcanol. Geotherm Res., 3, 1-37, 1978.

Spera, F., Some numerical experiments on the withdrawal of magma from crustal reservoirs, J. Geophys. Res., 89, 8222-8238, 1984.

Spera, F., D. Yuen, J. Greer, and G. Sewell, Dynamics of magma withdrawal from stratified magma chambers, Geology, 14, 723-726, 1986.

Tait, S., C. Jaupart, and S Vergniolle, Pressure, gas content and eruption periodicity of a shallow, cristallising magma chamber, Earth Planet. Sct Lett, 107-123, 1989.

Temam, R, Sur l'approximation de la solution des équations de NavierStokes par la méthode des pas fractionaries, Arch. Rat. Mech. Anal. $32,135-153,1969$.

Touloukian, Y.S., W.R. Judd, and R.F. Roy, Physical Properties of Rocks and Minerals, vol. 1, 548 pp., McGraw-Hill, New York, 1981.

Trial, A.F., F. Spera, J. Greer, and D.A. Yuen, Simulations of magma withdrawal from compositionally zoned bodies, J. Geophys. Res., 97, 6713-6733, 1992.

Vergniolle, S., and C. Jaupart, Separated two-phase flow and basaltic eruptions, J. Geophys. Res., 91, 12842-12860, 1986.

Wilson, L., R.S.J. Sparks, and G.P.L Walker, Explosive Volcanic Eruptions, IV, The control of magma properties and conduit geometry on eruption conduit behavior, Geophys. J. R. Astron. Soc., 63, 117$148,1980$.

Zienkiewicz, O.C., K. Morgan, B.V.K. Satya Sai, R. Codina, and M. Vázquez, A general algorithm for compressible and incompressible flow, II, Tests on the explicit form, Int. J. Numer. Methods Fluids, 20, $887-913,1995$.

A. Folch and J. Martí, Institute of Earth Sciences "Jaume Almera", CSIC, Lluís Solé Sabarís, 08028 Barcelona, Spain. (e-mail: afolch@paleo.jja.csic.es; j.marti@paleo.ija.csic.es.)

R. Codina and M. Vázquez, International Center for Numerical Methods in Engineering, Universitat Politècnica de Catalunya, Gran Capità s/n, 08034 Barcelona, Spain. (e-mail: codina@ulises.upc.es; mvazquez@ulises.upc.es.)

(Received November 12, 1997; revised March 26, 1998; accepted May 11, 1998.) 